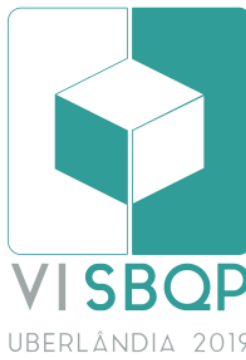

\title{
PROJETO COMPUTACIONAL E PERSONALIZAÇÃO DE LAYOUTS: UM MAPEAMENTO DA LITERATURA
}

\author{
LEITE, Raquel Magalhães \\ Universidade Estadual de Campinas, e-mail: raquelmleite@gmail.com \\ CELANI, Gabriela \\ Universidade Estadual de Campinas, e-mail: celani@unicamp.br
}

\begin{abstract}
RESUMO
A necessidade de personalizar espaços, principalmente nas camadas mais internas de um edifício, evolui segundo as mudanças de demandas dos usuários. A adoção de estratégias de projeto computacional por arquitetos e designers pode contribuir para que o agenciamento desse processo pelos sujeitos gere soluções de qualidade. Este trabalho é parte de uma pesquisa de mestrado em andamento e tem como objetivo desenvolver um panorama das aplicações de projeto computacional, personalização e automatização de layouts. Os exemplos identificados em publicações da última década são caracterizados de acordo com as camadas de intervenção, os agentes envolvidos e os momentos dessas proposições. Este estudo tem caráter exploratório e adota a estratégia do Mapeamento Sistemático da Literatura. Os resultados apontam um crescimento nas pesquisas sobre o tema, com uma maior tendência de estudos sobre a disposição de ambientes que sobre a organização de móveis em espaços internos. Como principais contribuições, são identificadas áreas potenciais para conduzir novos trabalhos, como reconfiguração e customização dimensional de layouts de mobiliário, combinados a processos conduzidos pelos usuários.
\end{abstract}

Palavras-chave: Layout, Projeto computacional, Personalização, Automatização.

\begin{abstract}
The need to customize spaces evolves according to the changing demands of users, especially in the inner layers of a building. Computational design strategies adopted by architects and designers enable the generation of adequate solutions with user agency. The present work is part of an ongoing master's degree research and aims to develop an overview of computational design applications, personalization and automation of layouts. Examples found in publications from the last decade were characterized according to their layers, agents and moments of intervention. This work has an exploratory character and develops a Systematic Mapping Study as research strategy. Results point to the growth in interest on the subject, with a greater number of studies on space planning than on furniture arrangement in internal spaces. The main contribution is the identification of potential topics for conducting new studies, such as reconfiguration and dimensional customization of furniture layouts, combined to user-driven processes.
\end{abstract}

Keywords: Layout, Computational design, Customization, Automation.

\section{INTRODUÇÃO}

O desenvolvimento de soluções para espaços internos ocorre, geralmente, de maneira improvisada (BRAND, 1994). Por razões diversas, principalmente nas tipologias mais cotidianas, como habitação e locais de trabalho, não existe o

LEITE, R. M.; CELANI, G. Projeto computacional e personalização de layouts: um mapeamento da literatura. In: SIMPÓSIO BRASILEIRO DE QUALIDADE DO PROJETO NO AMBIENTE CONSTRUÍDO, 6., 2019, Uberlândia. Anais... Uberlândia: PPGAU/FAUED/UFU, 2019. p 760-770. DOI https://doi.org/10.14393/sbqp19070. 
hábito de contratar profissionais para projetar e modificar espaços (BRAND, 1994; CAU/BR, 2015). Essa situação se agrava com a crescente perda de controle pelos usuários, em virtude da padronização de móveis industrializados diante de espaços cada vez mais reduzidos e da adoção de soluções de projeto que são personalizadas, mas na maioria das vezes não são flexíveis (FOLZ, 2008).

Um estudo de Duffy e Henney (1989) a respeito dos custos de um edifício ao longo de cinquenta anos demonstrou que, em geral, despende-se três vezes mais com modificações no edifício que com sua construção inicial. Além disso, esses autores apontam que a parcela mais substancial dos gastos ocorre em reformas na disposição dos ambientes. Comparativamente à manutenção de estruturas e serviços, o que se acumula com reestruturação de espaços ao longo desse período equivale a praticamente metade dos gastos totais (DUFFY; HENNEY, 1989).

A compreensão do edifício em camadas revela-se importante para verificar a frequência de modificações ao longo do tempo. Brand (1994) identifica seis camadas em um edifício (6S): site, structure, skin, services, space plan e stuff. Esse ordenamento se relaciona à variável temporal - site (ou terreno) perdura por gerações, ao passo que elementos na camada de stuff (ou objetos) podem ser modificados diariamente. Além disso, em cada camada, Brand (1994) diferencia os agentes responsáveis pelas mudanças. No caso de space plan e stuff, que configuram o universo do layout, esse papel é exercido por usuários, arquitetos e designers.

O desenvolvimento de alternativas de layout de maneira compartilhada entre arquitetos e usuários pode ser automatizado pelo projeto computacional. Pela compreensão do projeto como uma rede de informações estruturadas (MITCHELL, 1975), a inserção de parâmetros pelos usuários repercute na geração de uma gama de soluções, que depois podem ser otimizadas para determinadas situações. Nesse contexto, é possível obter variedade na personalização dos projetos sem que haja aumento significativo nos custos (KOLAREVIC, 2005).

Percebe-se, contudo, que a perspectiva da otimização se refere a um momento específico, ainda que para um conjunto de cenários possíveis. Quando são consideradas as mudanças agenciadas pelos usuários, um projeto otimizado passa a ser aquele que leva em conta a variabilidade de cenários (BRAND, 1994) e adaptabilidade ao longo do tempo (SCHMIDT III; AUSTIN, 2016), sem deixar de incorporar critérios de qualidade. Esse planejamento abrange, portanto, não apenas a pré-configuração de espaços, como também a sua reconfiguração (SCHMIDT III et al., 2010).

Diante desse contexto, questiona-se nesta pesquisa: quais estratégias de projeto computacional têm sido propostas para a personalização de layouts? Em quais escalas e momentos elas ocorrem e com que frequência incorporam agentes variados?

Este artigo tem como objetivo realizar uma caracterização das aplicações de projeto computacional, automatização e personalização em layouts, a fim de compreender quais os procedimentos utilizados, em quais momentos esses processos acontecem - antes ou após a ocupação do espaço - e se há envolvimento dos usuários. Além disso, pretende-se verificar quais são os 
elementos selecionados para personalização em cada caso e identificar se há correlações entre as camadas de intervenção.

\section{METODOLOGIA}

Este estudo possui caráter exploratório e caracteriza-se como pesquisa bibliográfica. O procedimento metodológico adotado é o Mapeamento Sistemático da Literatura (MSL). Segundo James, Randall e Haddaway (2016), a condução de um MSL possibilita obter um panorama a respeito do tema que se deseja estudar, classificar resultados encontrados segundo critérios de interesse e identificar lacunas para a condução de novas pesquisas. A sistematização de um protocolo possibilita atingir um amplo espectro de publicações e evitar viés.

A estratégia utilizada nesta pesquisa baseia-se em Ruiz e Granja (2013) e organiza-se em dez etapas:

a) Identificação da problemática e definição da questão de pesquisa, já explicitadas na introdução;

b) Definição dos termos de busca: os termos extraídos diretamente da questão de pesquisa são "layout", "projeto computacional" e "personalização". Acrescentou-se também a palavra-chave "automatização", por se relacionar diretamente ao tema;

c) Seleção das bases de dados: as buscas ocorreram nas bases internacionais Web of Science, Scopus, Avery e Cumulative Index of Computer-Aided Architectural Design (CumInCAD);

d) Formulação de strings: os termos foram traduzidos para inglês e combinados para pesquisa nas bases. As três strings de busca foram layout AND "computational design", layout AND automat* e layout AND (customiz* OR customis*);

e) Definição de critérios de seleção dos artigos: foram selecionadas publicações de periódicos e de conferências entre 2008 e 2018 nas áreas de arquitetura, estudos do ambiente e tecnologia da construção;

f) Organização dos dados: os dados foram transferidos para um gerenciador de referências para exclusão de duplicatas e de artigos não aderentes ao tema. Foram mantidas as publicações relacionadas à área da arquitetura e às camadas de space plan e stuff (BRAND, 1994);

g) Adição de artigos: foram acrescentados artigos publicados no último congresso da Sociedade Iberoamericana de Gráfica Digital (SIGraDi), os quais ainda não haviam sido indexados pela base CumlnCAD;

h) Extração de sistematizada de dados: inicialmente realizou-se uma caracterização segundo aspectos bibliométricos, na qual os artigos foram classificados por ano de publicação. Em seguida, após a leitura dos resumos e a busca de informações nos artigos completos, essa categorização foi refinada e os dados foram filtrados de acordo com a pergunta de pesquisa. A leitura possibilitou também a identificação de alguns trabalhos que não se inseriam nas camadas de space plan e stuff, os quais foram removidos do corpus de análise. Nessa etapa, buscou-se fazer uma categorização segundo os componentes 
escolhidos para personalização, a estratégia utilizada, o momento da intervenção e os agentes envolvidos no processo;

i) Análise dos dados filtrados: por meio da organização em planilhas e da subsequente tradução das informações em gráficos, foi possível visualizar de maneira mais clara as tendências e lacunas na área de pesquisa delimitada;

j) Conclusões, apresentadas ao final do artigo.

\section{RESULTADOS E DISCUSSÃO}

A primeira etapa da análise de dados se relaciona aos aspectos bibliométricos. A Figura 1 ilustra a evolução dos dados durante as etapas (f), (g) e (h) deste MSL. O somatório dos resultados encontrados nas bases foi de 260 publicações. Após a remoção de duplicidades, a filtragem foi realizada em dois ciclos. Inicialmente, por meio da leitura dos títulos, atribuíram-se os marcadores "sim", "talvez" e "não" para cada publicação, o que identificou 73 artigos com possível aderência. No segundo ciclo, após a leitura e filtragem dos resumos dos artigos identificados com "talvez", restaram 54 publicações aderentes. Por fim, adicionaram-se mais 4 artigos identificados no último congresso da Sociedade Iberoamericana de Gráfica Digital (SIGraDi), o que configurou o total de 58 publicações.
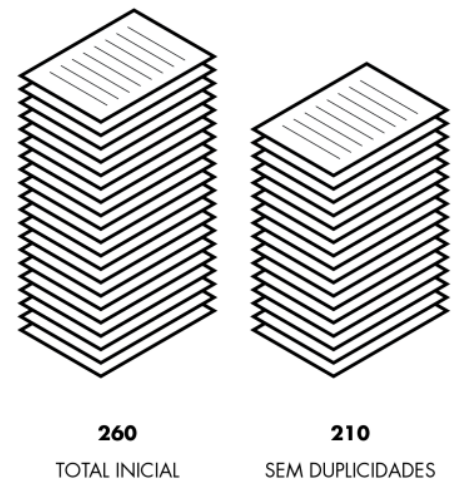

SEM DUPLICIDADES

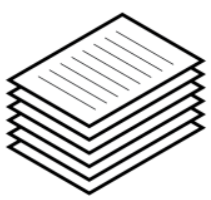

73

CICLO 1: SIM+TALVEZ

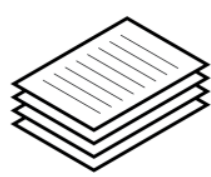

54

CICLO 2: SIM

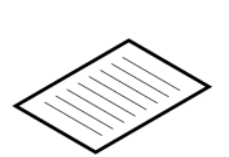

4

ADICCÃO

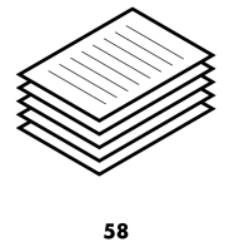

TOTAL FINAL

Figura 1 - Evolução da quantidade de resultados ao longo do MSL Fonte: Autoras (2019)

Em seguida, os 58 artigos foram classificados por ano de publicação (Figura 2). O gráfico mostra o crescimento das pesquisas a respeito do tema, com uma lacuna em 2012. Observa-se que mais de $70 \%$ das publicações ocorreram nos últimos cinco anos, sendo 2018 o ano que apresentou mais resultados.

A extração sistematizada de dados foi realizada após a organização das informações em planilhas. As respostas para a pergunta de pesquisa foram procuradas nos resumos e, quando necessário, diretamente nos textos dos artigos completos. Nessa fase, foram identificados três trabalhos de revisão da literatura, os quais foram removidos do corpus de análise por trazerem panoramas com diversos exemplos e não uma proposta ou análise específica. Desse modo, restaram 55 publicações com aplicações desenvolvidas sobre projeto computacional e personalização de layouts. 
Os elementos selecionados para automatização são variáveis, o que ilustra a amplitude do termo layout em arquitetura. Para essa categorização, foram consideradas as duas camadas de Brand (1994) que se relacionam ao espaço interno de um edifício: space plan e stuff. Como elucida a Figura 3, a maioria dos artigos, quando trata de layout, refere-se a space plan, que representa a distribuição e a articulação dos ambientes (46 publicações). Em segundo lugar, vem a camada stuff, que em geral contempla o mobiliário (12 publicações).

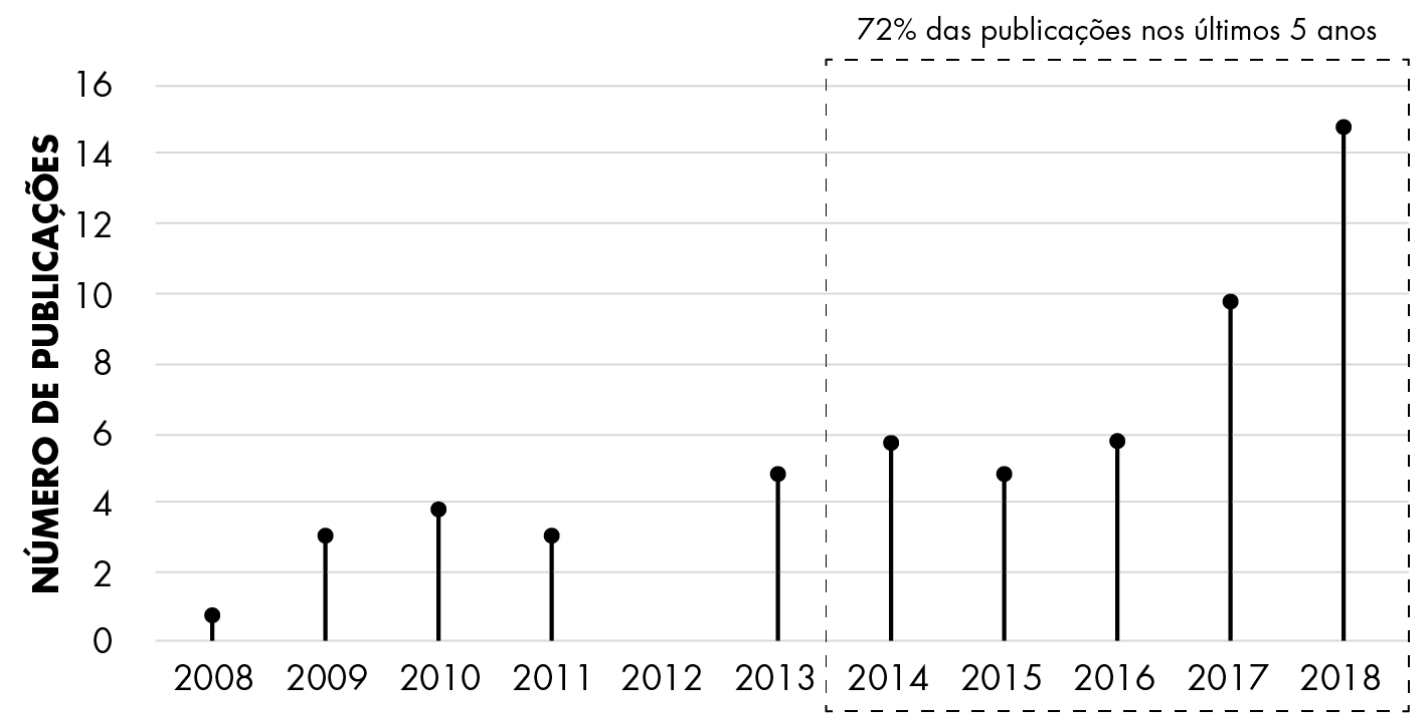

Figura 2 - Número de publicações por ano -

Fonte: Autoras (2019)

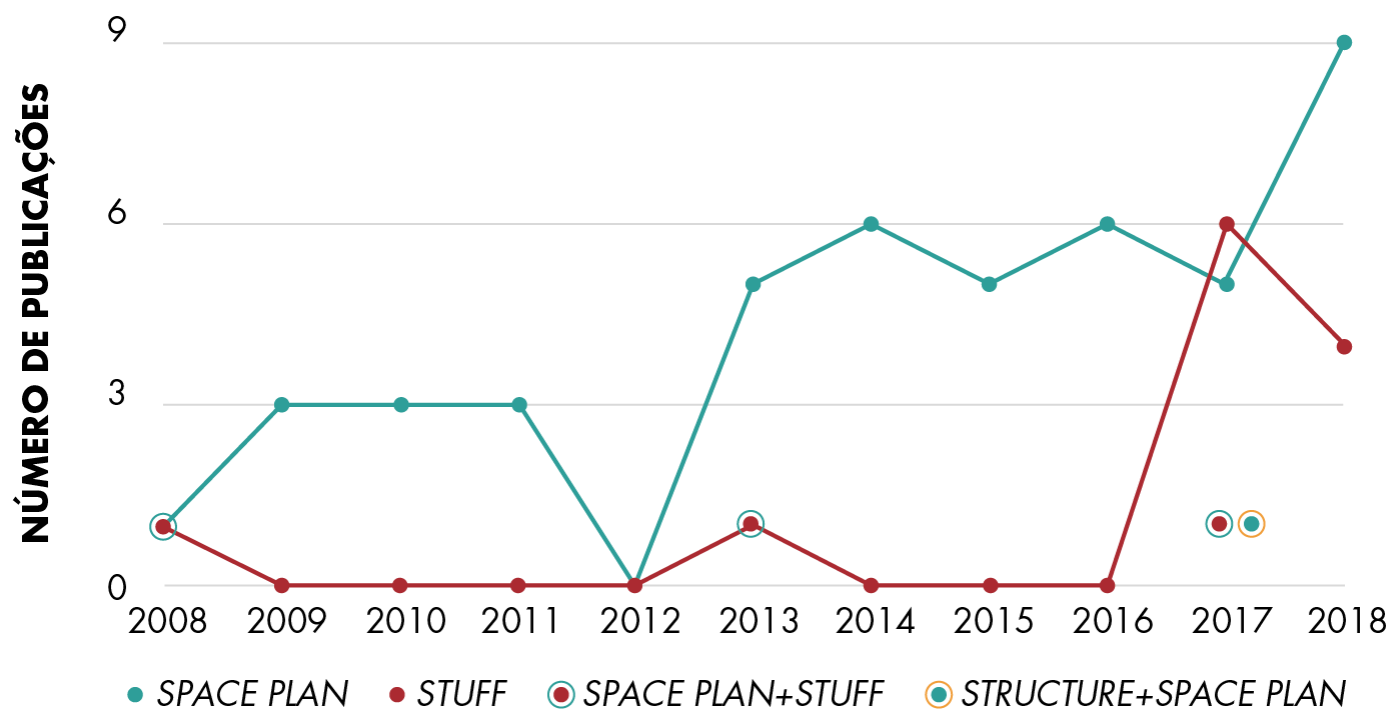

Figura 3 - Quantidade de publicações por ano em cada camada ou combinação de camadas sistematizadas por Brand (1994) Fonte: Autoras (2019)

Quatro trabalhos abordam mais de um componente ao mesmo tempo, considerando a relação entre camadas. Sobre a junção de space plan e structure, um artigo trata da distribuição de vedações internas articulada à localização de pilares (STEINER et al., 2017). Além disso, outros três artigos 
exploram de maneira conjunta a organização de ambientes (space plan) e a distribuição de objetos (stuff) (HOSOKAWA et al., 2008; NAGY et al., 2017; SUTER, 2013). Nessas três abordagens, o arranjo dos ambientes se dá pelo posicionamento das vedações internas e, em seguida, os móveis são distribuídos de acordo com a posição das paredes. Não se explorou, portanto, uma perspectiva integrada de vedação e mobiliário que considerasse a utilização da mobília como elemento de conformação do espaço.

Outro ponto importante nessa categorização é o aspecto escolhido para automatização dentro de cada camada. Por exemplo, a personalização de layouts de mobiliário na prática mercadológica ocorre, muitas vezes, por meio de projetos sob medida. Contudo, todos os artigos deste mapeamento que contemplam a mobília tratam do posicionamento de objetos com dimensões pré-definidas em um espaço. Não se considera a variação dimensional desses móveis, o que remete, por um lado, à utilização de mobiliário padronizado, produzido pela indústria de massa e, por outro, a uma possibilidade de reaproveitamento de móveis durante uma mudança de ambiente (YAMAKAWA et al., 2017).

tangible user interface

metropolis-hasting algorithm unified matrix

internet-based interface

interactive artificial selection

daylight simulation simulated annealing

network representations multi-agent topology

building energy simulation RFID user interface

evolutionary algorithms

BIM graphs space syntax

neural networks

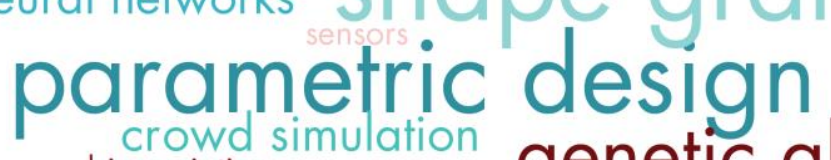

biosemiotics

program of requirements

hypergraphs

structured aggregation

design rules

distance field physical models

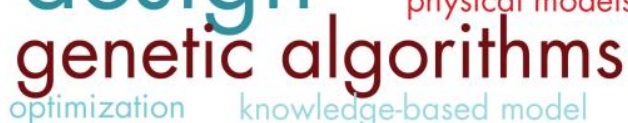

probabilistic model

Figura 4 - Estratégias de projeto computacional e personalização de layouts utilizadas nos trabalhos mapeados

Fonte: Autoras (2019)

Os procedimentos adotados para personalização e automatização de layouts foram variados e, muitas vezes, envolveram a combinação de mais de uma abordagem. A Figura 4 traz uma nuvem de palavras, na qual a dimensão dos termos reflete a quantidade de trabalhos em que cada estratégia foi empregada neste MSL. Aplicações de gramática da forma (9 trabalhos), projeto paramétrico ou associativo (7 trabalhos), algoritmos evolutivos (5 trabalhos) e algoritmos genéticos (4 trabalhos) foram recorrentes. Conforme aponta Oxman (2006), esses são alguns dos procedimentos consolidados na geração de soluções em arquitetura por meio de projeto computacional. Além disso, 10 artigos abordaram a integração de estratégias algorítmicas e generativas ao building information modeling (BIM). Essa combinação possibilita a tradução de alternativas de projetos para os componentes 
efetivos da construção, com estimativas de custos e geração de desenhos para execução (VELOSO; CELANI; SCHEEREN, 2018). Alguns outros procedimentos utilizados foram grafos (7 artigos), simulações baseadas em desempenho ambiental ou ocupação humana (9 artigos), sintaxe espacial (3 artigos) e machine learning (3 artigos).

Com relação ao momento em que ocorre a intervenção no layout, a Figura 5 ilustra que há três vezes mais trabalhos que fazem propostas de préconfiguração (36) que de reconfiguração (12), sendo que 5 deles trazem aplicações que se adequam aos dois momentos. Os agentes envolvidos, por sua vez, consideram sempre a figura do projetista, predominantemente o arquiteto. Pouco mais da metade dos artigos apresenta intervenções em layouts comandadas apenas pelos projetistas (28), e 23 levam em conta também a interferência dos usuários, em algum grau. Os demais trazem outros agentes, como programadores e fabricantes.

\section{MOMENTO DA INTERVENÇÃO}

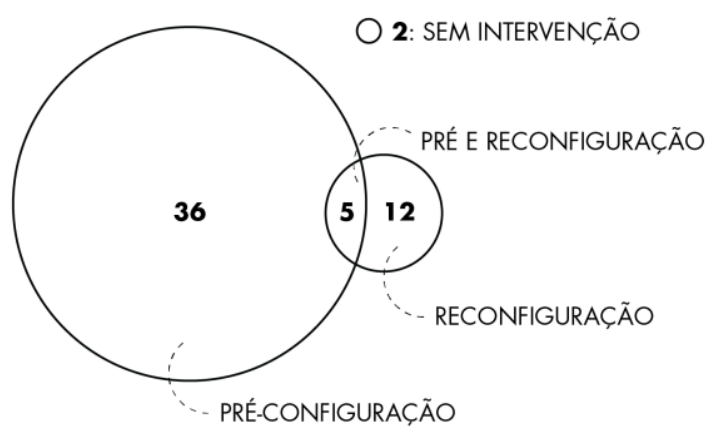

AGENTES DA INTERVENÇÃO

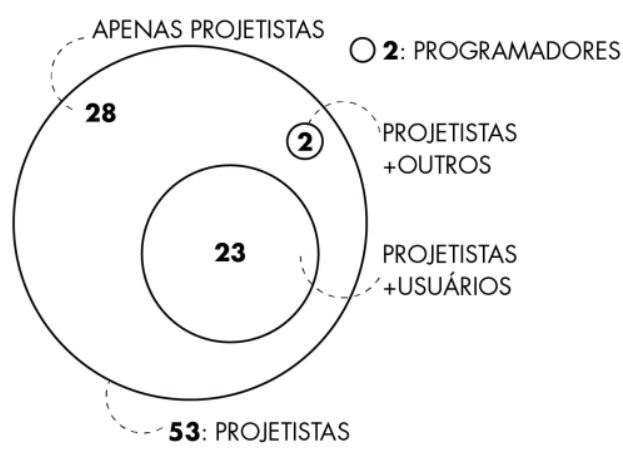

Figura 5 - Momentos em que há intervenção no layout e agentes envolvidos nesse processo

Fonte: Autoras (2019)

Como se observa na Figura 6, a análise cruzada de dados elucida algumas conexões entre a camada de layout, os agentes envolvidos e o momento da intervenção. Dos artigos que exploram a camada stuff, mais da metade considera o agenciamento do processo pelos usuários e se refere à reconfiguração, de maneira independente ou complementar à préconfiguração. Vale mencionar, também, que quase todos artigos foram publicados nos últimos dois anos (Figura 3). Em contrapartida, em space plan, apenas 1/5 dos trabalhos se dedica à reconfiguração, e menos da metade explora possibilidades de integração dos usuários no processo de projeto.

Como discutido por Duffy e Henney (1989), modificações na planta ao longo do tempo repercutem na parcela mais considerável de gastos do ciclo de vida de um edifício. O baixo índice de publicações sobre reconfiguração em space plan aponta uma lacuna para o desenvolvimento de novos estudos, sob a perspectiva sustentável de renovação do espaço construído existente.

Na Figura 6, percebe-se também que a inclusão dos usuários no processo de personalização de layouts ocorre, proporcionalmente, com maior intensidade, na camada dos objetos que na da organização de ambientes. Além disso, poucos trabalhos abordam a modificação de mais de uma camada ao mesmo tempo. Ainda assim, por mais que não tragam uma abordagem integrada, alguns artigos expressam a importância do diálogo entre camadas. 
Por exemplo, Anderson et al. (2018) desenvolveram um procedimento para a distribuição automática de mesas em escritórios, pela combinação entre algoritmos e BIM. A integração dos usuários dos espaços ao projeto se deu por meio de uma interface associada à plataforma BIM. Já o trabalho de Côco Júnior e Celani (2018) também traz a conexão entre essas duas estratégias, mas para a automatização de layouts de banheiros pré-fabricados. Nesse último caso, a pesquisa traz a perspectiva da integração entre arquitetos e fabricantes durante o desenvolvimento das alternativas de projeto.

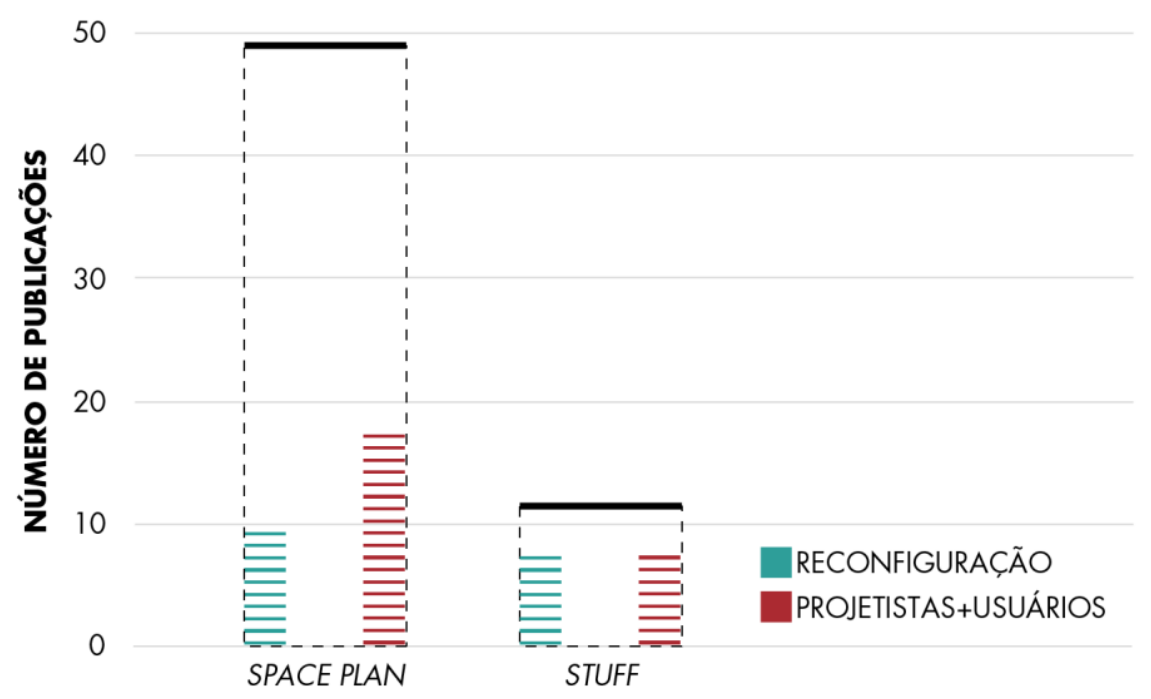

Figura 6 - Associação entre as camadas, os momentos e os agentes da intervenção Fonte: Autoras (2019)

Em resumo, os resultados demonstraram possíveis conexões entre procedimentos variados para personalização e automatização de layouts, os quais se integram também a outras ferramentas de projeto computacional que podem envolver no processo agentes distintos do ciclo de vida de um edifício. O layout de espaços, que transita entre a disposição de ambientes e de objetos, tem sido um objeto de estudos cada vez mais frequente e pode se beneficiar do diálogo entre camadas para a reestruturação de espaços existentes.

\section{CONCLUSÕES}

De maneira crescente, a inserção da tecnologia da informação no cotidiano das pessoas modifica a rotina de diversas profissões. Em arquitetura, o projeto computacional possibilita a experimentação de soluções e abre novas possibilidades para o agenciamento do processo de projeto pelos usuários, como por exemplo na configuração de layouts.

O desenvolvimento deste MSL possibilitou a identificação de algumas tendências, como o maior foco da compreensão do termo layout como organização dos ambientes, ou space plan, que como distribuição dos objetos, ou stuff, se consideradas as camadas de Brand (1994). Também foi reconhecido um campo potencial para estudos que tratem da associação entre as camadas space plan e stuff, como por exemplo pela personalização integrada de vedações e mobiliário. 
Além disso, na camada stuff, não foram encontradas evidências que considerem a variação dimensional dos objetos na definição do layout. Em geral, as pesquisas direcionam-se a automatizar o posicionamento de objetos com dimensões pré-definidas. Identifica-se, assim, um contexto oportuno para a condução de estudos sobre personalização de layouts em que os móveis possuam dimensões variáveis. Como indicado por Kolarevic (2015), a customização dimensional é um dos principais desafios na proposição de interfaces que dialoguem também com os usuários.

Averiguou-se também que um número reduzido de publicações prevê a integração dos usuários ao processo de personalização e à reconfiguração de espaços. A maioria das estratégias considera a elaboração de novos projetos por arquitetos e outros profissionais da área. Essa tendência revela um campo amplo para a realização de estudos sob a perspectiva da adaptabilidade, ou seja, da capacidade de um edifício maximizar seu valor ao longo do tempo por meio da acomodação contínua de mudanças (SCHMIDT III; AUSTIN, 2016). Essa acomodação envolve, justamente, as articulações entre uso e tecnologia para reconfiguração espacial (SCHNEIDER; TILL, 2007).

Pesquisas posteriores a este MSL podem expandir o escopo para mais bases de dados e intervalos de tempo. A amostragem também pode ser ampliada a partir das referências citadas nos trabalhos. Além disso, um aprofundamento nos conteúdos dos artigos pode contribuir para identificar correlações entre as estratégias de personalização empregadas e o papel dos diversos agentes no desenvolvimento dos projetos.

Por fim, os diversos estudos desenvolvidos sobre projeto computacional e personalização de layouts demonstram que a utilização das tecnologias de projeto digitais pode fornecer, por meio de estratégias diversas, alternativas à improvisação na organização de espaços. Por outro lado, essas tecnologias geram também a oportunidade de os projetistas agregarem a complexidade dos contextos sociais como informações de projeto. Há um campo propício para a realização de pesquisas que contemplem o ciclo de vida dos edifícios, especialmente com a incorporação dos usuários à prática arquitetônica.

\section{AGRADECIMENTOS}

O presente trabalho foi realizado com apoio da Coordenação de Aperfeiçoamento de Pessoal de Nível Superior - Brasil (CAPES) - Código de Financiamento 001.

\section{REFERÊNCIAS}

ANDERSON, C. et al. Augmented space planning: Using procedural generation to automate desk layouts. INTERNATIONAL JOURNAL OF ARCHITECTURAL COMPUTING, v. 16, n. 2, p. 164-177, jun. 2018.

BRAND, S. How Buildings Learn. New York: Penguin Books, 1994.

CÔCO JÚNIOR, V. H.; CELANI, G. From the automated generation of layouts to fabrication with the use of BIM: a new agenda for Architecture in the 21 st century. In: XXII CONGRESSO INTERNACIONAL DA SOCIEDADE IBEROAMERICANA DE GRÁFICA DIGITAL, 7 nov. 2018, São Carlos. Proceedings... São Carlos: Blucher Design Proceedings, 7 nov. 2018. p. 23-30. Disponível em: 
<https://www.proceedings.blucher.com.br/article-details/-29680>. Acesso em: 11 nov. 2018.

CONSELHO DE ARQUITETURA E URBANISMO DO BRASIL (CAU/BR). Pesquisa

CAU/BR Datafolha. Disponível em: <https://www.caubr.gov.br/pesquisa2015/>. Acesso em: 13 mar. 2019.

DUFFY, F., HENNEY, A. The Changing City. London: Bullstrode, 1989.

FOLZ, R. R. Projeto tecnológico para produção de habitação mínima e seu mobiliário. 2008. Tese (Doutorado em Arquitetura e Urbanismo) - Escola de Engenharia de São Carlos, Universidade de São Paulo, São Carlos, 2008. Disponível em: <http://www.teses.usp.br/teses/disponiveis/18/18141/tde06082008-100756/>. Acesso em: 22 jan. 2019.

HOSOKAWA, T. et al. Tangible Design Support System Using RFID Technology. In: TEI'08 - INTERNATIONAL CONFERENCE ON TANGIBLE AND EMBEDDED INTERACTION, 2008, 2, Bonn. Proceedings... Bonn: [s.n.], 2008. p. 75-78.

JAMES, K. L.; RANDALL, N. P.; HADDAWAY, N. R. A methodology for systematic mapping in environmental sciences. Environmental Evidence, v. 5, n. 1, p. 7, 26 abr. 2016.

KOLAREVIC, B. (Org.). Architecture in the Digital Age. 1st. ed. New York: Taylor \& Francis, 2005.

KOLAREVIC, B. From Mass Customisation to Design 'Democratisation'. Architectural Design, v. 85, n. 6, p. 48-53, 2015.

MITCHELL, W.J. The Theoretical Foundation of Computer-Aided Architectural Design. Environment and Planning B: Planning and Design, v. 2, n. 2, p. 127-150, 1 dez. 1975.

NAGY, D. et al. Project Discover: An Application of Generative Design for Architectural Space Planning. In: SYMPOSIUM ON SIMULATION FOR ARCHITECTURE AND URBAN DESIGN, 2017, Toronto. Proceedings... Toronto: Simulation Councils, 2017. p. 59-66.

OXMAN, R. Theory and design in the first digital age. Design Studies, Digital Design. v. 27, n. 3, p. 229-265, 1 maio 2006.

RUIZ, J.A., GRANJA, A.D. Um Mapeamento Sistemático da Literatura sobre a relação entre valor e colaboração na construção. In: SIMPÓsIO BRASILEIRO DE GESTÃO E ECONOMIA DA CONSTRUÇÃO, 2013, 8, Salvador. Anais... Salvador, 2013.

SCHMIDT III, R. et al. What is the meaning of adaptability in the building industry? In: INTERNATIONAL CONFERENCE ON OPEN AND SUSTAINABLE BUILDING, 16, 2010, Bilbao. Proceedings... Bilbao: Labein Tecnalia, 2010. p. 233242.

SCHMIDT III, R.; AUSTIN, S. Adaptable Architecture: theory and practice. 1st. ed. New York: Routledge, 2016.

SCHNEIDER, T.; TILL, J. Flexible Housing. 1st. ed. Oxford: Architectural Press, 2007. STEINER, B. et al. Integrated Structural-Architectural Design for Interactive Planning. Computer Graphics Forum, v. 36, n. 8, p. 80-94, 2017. 
SUTER, G. Structure and Spatial Consistency of Network-Based Space Layouts for Building and Product Design. CAD Computer Aided Design, v. 45, n. 8-9, p. 1108-1127, 2013.

VELOSO, P.; CELANI, G.; SCHEEREN, R. From the Generation of Layouts to the Production of Construction Documents: An Application in the Customization of Apartment Plans. Automation in Construction, v. 96, p. 224-235, dez. 2018.

YAMAKAWA, T. et al. Computer Simulation of Furniture Layout When Moving from One House to Another. In: SCCG 2017 - SPRING CONFERENCE ON COMPUTER GRAPHICS, 2017, 33, Mikulov. Proceedings... Mikulov: Association for Computing Machinery, 2017. 